\title{
Ernst Bonek als Wissenschaftler und Lehrer
}

\author{
C. Mecklenbräuker
}

Online publiziert am 15. November 2012

(C) Springer-Verlag Wien 2012

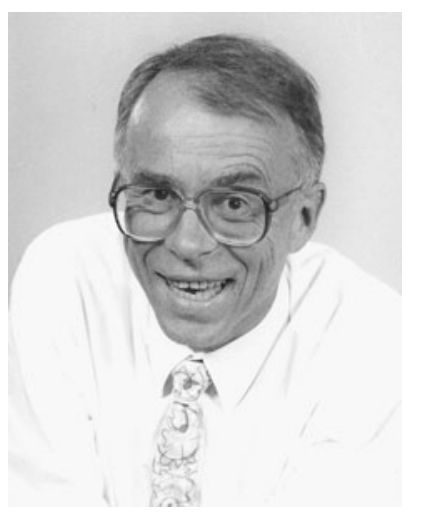

Ernst Bonek
Über den Sportler Ernst Bonek und den Entrepreneur in ihm ist an anderer Stelle schon berichtet worden. An dieser Stelle soll der Lehrer und Wissenschaftler vorgestellt werden.

Etwas beschämt blätterte ich anlässlich des Informationstechnischen Kolloquiums in den Unterlagen zur Vorlesung "Wellen und Leitungen" (3. Auflage, 1986) verfasst von Gottfried Magerl und Ernst Bonek. In vielerlei Hinsicht wird den Studentinnen und Studenten heute nicht mehr diese analytische Tiefe im Stoff vermittelt. Aber alles der Reihe nach. Lassen Sie mich anfangen mit einer Zusammenfassung der wesentlichen Meilensteine seiner Laufbahn.

Ernst Bonek wurde Mitte Februar 1942 in Wien geboren. Er studierte Nachrichtentechnik und diplomierte 1966 mit Auszeichnung an der damaligen Technischen Hochschule. Wissenschaftlich beschäftigte er sich intensiv mit Transportphänomenen in III-VHalbleitern unter der Anleitung von Professor Herbert König. Vielleicht übte aber sein Freund und Mentor Prof. Hans Pötzl in dieser Zeit bereits größeren Einfluss auf inn aus. Im Jahr 1972 promovierte er zum Dr. techn. mit Auszeichnung mit der Dissertation "Leitfähigkeit von III-V-Halbleitern im Gebiet heißer Elektronen - eine Untersuchung bei 4-mm-Wellen". Anschließend wechselte er auf das Gebiet der Laser und habilitierte sich mit seiner Schrift "Millimeterwellen - Modulation von Kohlendioxid-Lasern" während 1975/1976 für das Fach Hochfrequenztechnik. Im Jahr 1978 wurde er zum AuBerordentlichen Professor ernannt.

Während der beiden Jahre 1980/1981 war er Konsulent der ESTEC in Noordwijk in den Niederlanden. Es ging um Satellitenkommunikation, aber nicht zwischen Erde und Satellit, sondern zwischen Satelliten untereinander. Während dieser Zeit begegnete ich Ernst Bonek erstmals. Das war im Herbst 1980. Ich erinnere mich noch an seinen Besuch in Eindhoven zum Abendessen, der den Umzug der Familie Mecklenbräuker von Eindhoven nach Wien bereits vorzeichnete. Ernst Bonek schrieb meinen Eltern einen langen Brief, in dem er die schulischen Gegebenheiten in Wien genauestens beschrieb und beeinflusste damit meinen weiteren Schulweg.

1984 wurde er zum Ordinarius für Hochfrequenztechnik an die inzwischen umbenannte Technische Universität Wien berufen.

Prof. Ernst Bonek ist der maßgebliche Initiator von Forschung und Lehre auf dem Gebiet des Mobilfunks in Österreich und hat diese Disziplin seit 1984 am Institut für Nachrichtentechnik und Hochfrequenztechnik an der Fakultät für Elektrotechnik und Informationstechnik der TU Wien in einer international führenden For- schungsgruppe etabliert. Seit 1988 kooperierte er auf diesem Gebiet intensiv mit der österreichischen Post und Telegraphenverwaltung.

Er ist der Initiator des Forschungszentrums Telekommunikation Wien, dessen Vizepräsident er von 1999 bis 2002 war. Aus Ernst Boneks Schule kommt eine ganze Generation von hervorragenden Technikerinnen und Technikern der mobilen Kommunikation.

Am 30. Mai 2008 erhielt er das Ehrendoktorat der Universität Lund: Ernestus Bonek, professor technicae radiofrequentialis Vindobonensis, communicationum mobilium auctor et fautor.

Ernst Bonek hat sich seit 1982 mit Sicherheitsaspekten im Mobilfunk auseinandergesetzt, insbesondere zur Festsetzung von Grenzwerten für elektromagnetische Felder. Er überzeugte die Mitglieder des zuständigen österreichischen Gremiums, die Grundsätze von ICNIRP zu übernehmen, weil diese auf wissenschaftlichen Erkenntnissen beruhen.

Eine seiner wissenschaftlichen Maxime war stets: „Measure before you model." Ende der 1990er Jahre war unter theoretisch orientierten Forschern die Ansicht weit verbreitet, die Eigenschaften des Mobilfunkkanals seien vollständig erforscht. Messungen auf diesem Gebiet seien nun nicht mehr erforderlich. Diese Ansicht wurde gründlich überholt durch die sich rasant entwickelnde Forschung auf dem Gebiet der MIMO-Übertragungsverfahren, bei denen auf beiden Seiten des Mobilfunkkanals Antennengruppen verwendet werden. In Ernst Boneks Schule entstanden einige bedeutende Arbeiten zur Charakterisierung von MIMO-Kanälen im Mobilfunk.

Er förderte Englisch sehr als Unterrichtssprache an der TU Wien, und die fast schon legendäre Lehrveranstaltung „How to publish a scientific paper", die er seit vielen Jahren gemeinsam mit Walter Leeb abhielt, erfreute sich hoher Beliebtheit - auch wegen der geradezu kabarettistischen Einlagen. Stets betonte er, dass die Resultate und Produkte aus der Arbeit einer Ingenieurin/eines Ingenieurs am Markt bestehen müssen.

Seit vielen Jahren ist Ernst Bonek in der Volksbildung engagiert sowie im Wiener Ableger der Skeptiker (Gesellschaft zur wissenschaftlichen Untersuchung der Parawissenschaften e. V.). Zu den Zielen der Skeptiker gehört Aufklärung über Pseudowissenschaft und Scharlatanerie, eine Festigung des skeptisch-naturwissenschaftlichen Weltbildes und die wissenschaftliche Überprüfung parawissenschaftlicher Behauptungen. Besonders stört die Skeptiker, dass in Österreich sogar im Parlament die Wünschelrutengänger unterwegs sind. „Da muss man dagegenhalten", fordert Ernst Bonek. Und ich kann ihm nur beipflichten.

Er referiert bei vielen Gelegenheiten über die gesundheitlichen Auswirkungen elektromagnetischer Felder und legt hierbei großen Wert auf eine genaue Darstellung des jeweils neuesten Stands der Forschung auf diesem Gebiet. Er legt dabei Wert auf die Feststellung, dass elektrosensible Personen unbedingt ernst genommen

Mecklenbräuker, Christoph, Univ.-Prof. Dipl.-Ing. Dr.-Ing., Institute of Telecommunications, Technische Universität Wien, Gußhausstraße 25, 1040 Wien, Österreich (E-Mail: christoph.mecklenbraeuker@tuwien.ac.at) 
werden müssen. In Versuchen leiden sie tatsächlich, wenn sie die Wirkung elektromagnetischer Felder bewerten müssen, denen sie gar nicht ausgesetzt waren. Nocebo-Effekt nennt das die Wissenschaft.
Vor allem lehrt er uns jedoch Demut vor der inhaltlichen Kritik, deren einzige Aufgabe es ist, die Qualität der Ergebnisse zu sichern - respektlos vor der bestehenden Hierarchie.

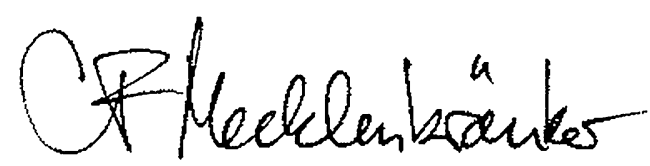

